\title{
The Effect of School Nutritionists' Self-efficacy and Ego-resilience on Their Organizational Commitment
}

\author{
Mi Jung Kim \\ Mindfulness EFT Psychological Counseling Research Center, 590-7, Munbok-ro, \\ Sannae-myeon, Gyeongju-si, Gyeongsangbuk-do, Republic of Korea \\ kmj5044@naver.com
}

\begin{abstract}
This study examined the effects of nutritionists' self-efficacy and ego-resilience on organizational commitment. Surveys were conducted against 108 school nutritionists in elementary, middle, and high schools in K-city and U-city from 15 April to 30 April 2019. Self-efficacy, ego-resilience, and organizational commitment scales were used as research tools, and PASW 22.0 for Windows was used for data processing. The results of the study were as follows: First, self-efficacy, ego-resilience, and organizational commitment as perceived by school nutritionists were found to have significant correlations. Second, selfefficacy and ego-resilience perceived by school nutritionists affected organizational commitment. This study is significant in that it provides political measures and suggestions regarding school nutritionists, who are nutrition experts that can support healthy school life, by verifying how the self-efficacy and ego-resilience of nutritionists can improve organizational commitment levels.
\end{abstract}

Keywords: Self-efficacy, Ego-resilience, Organizational commitment, Nutritionist, School

\section{Introduction}

Because adolescents are transitioning from childhood to adulthood, they have many physical developmental characteristics, and as it is an important time for many physical changes, they must maintain a balanced nutritional state based on good dietary habits [1]. In addition, it is necessary to form desirable dietary habits and a balanced nutritional state during adolescence, because habits formed in adolescence are difficult to change once you reach adulthood [2]. However, living standards in Korea have changed recently with the changes in family structures, the social participation of women, and the increase in national income, bringing about the emergence of convenience-focused culinary culture. This resulted in poor dietary habits in adolescents, such as increased consumption of processed or fast foods and indiscriminate and excessive consumption of nutrients, leading to a serious nutritional imbalance [3].

In addition to these socio-environmental characteristics, expectations for nutrition education with professional nutritional information are increasing in the face of an indiscriminate supply of various health foods through mass media and the flood of unaccounted health-related information. Since the School Meals Act was enacted in Korea in 1981, school meals were provided in all elementary, middle, and high schools nationwide

Article History:

Received (November 19, 2019), Review Result (December 23, 2019), Accepted (January 27, 2020) 
until 2003, achieving a quantitative growth of school meals. As the importance of school meals has been highlighted with the expansion of school meals, there has also been an increase in the consumers' interests and demands regarding the overall quality of school meals and nutrition management, including services related to school meals, as well as ingredients and foods provided in the school meals. There can be social conflicts between workers in different positions within the school nutritionist organization, due to the difference in perception. Such conflicts within the organization become a factor of job stress for the members and can be an important variable that can affect the behavior and attitude of workers in the organization.

On the other hand, one way to overcome this problem is through self-efficacy, as selfefficacy acts as a variable that greatly affects the execution of problem-solving when faced with a new environment. Existing studies show that when the conflict within the organization gets bigger, it harms organizational commitment, and the improvement of self-efficacy has meaningful relations in overcoming such negative effects [4][5].

Organizational commitment refers to the affection for the workplace; the psychological attachment the member feels toward the organization, or the positive attitude and tendency to fulfill organizational goals [6][7][8]. There have been accumulating studies recently on the organizational commitment of teachers in schools [9][10][11][12]. Therefore, it is necessary to review the variables that affect the organizational commitment of nutritionists, as well as to improve the work environment to improve the organizational commitment of school nutritionists. Different variables have been suggested to positively affect the organizational commitment of nutritionists, but above all, the concept of ego-resilience as an internal variable of the nutritionist has emerged as a new perspective for explaining and analyzing the commitment behavior of teachers [13][14]. Recently, there have been various studies on egoresilience as a factor that affects organizational commitment in public servants and education [15][16][17]. In [15]'s study, ego-resilience played the role of a mediating variable between the job stress of regional public servants and their organizational commitment, having a positive effect on organizational commitment. Therefore, this study seeks to analyze the effect of the relationship between self-efficacy, ego-resilience, and organizational commitment through existing studies. The following research questions were selected to achieve the purpose of the study.

The following research questions were selected to achieve the purpose of the study.

First, what is the relationship between self-efficacy, ego-resilience, and organizational commitment of school nutritionists?

Second, what is the effect of school nutritionists' self-efficacy and ego-resilience on their organizational commitment?

\section{Research method}

\subsection{Research subject}

Surveys were conducted against 121 school nutritionists in elementary, middle, and high schools in K-city and U-city from 15 April to 30 April 2019. After excluding negligent responses, a total of 108 questionnaires were processed statistically and used for analysis.

\subsubsection{General background of research subjects}

Upon examining the general background of the research subjects, there was the highest percentage of nutritionists in their 40 s with 59 respondents $(54.2 \%)$, and in experience, the 
highest was more than 5 years and less than 10 years with 42 respondents (38.4\%). The highest school type was an elementary school with 80 nutritionists $(74.2 \%), 66$ respondents worked in schools with more than 5 less than 10 workers cooking $(61.1 \%)$, and 58 respondents worked in schools with less than 1000 students who were provided meals $(53.2 \%)$.

\subsection{Research tool}

\section{1) Self-efficacy}

In this study, the nutritionists' self-efficacy scale was completed with references to existing studies [18]. This scale consists of 25 questions on the sub-factors of general self-efficacy and personal self-efficacy. In the reliability coefficient of the self-efficacy scale, total self-efficacy was .92 , general self-efficacy was .91, and personal self-efficacy was .95 .

2) Ego-resilience

The ego-resilience scale of [19] was used as the tool to measure the ego-resilience of nutritionists. The scale of ego-resilience consists of 29 questions on the following four subfactors: confidence, the efficiency of interpersonal relations, optimistic attitude, and anger control. In the reliability coefficient of the ego-resilience scale, total ego-resilience was .64, confidence was .86 , the efficiency of interpersonal relations was .85 , the optimistic attitude was .84, and anger control was .64.

3) Organizational commitment

This study uses the questionnaire modified and translated by [20] to measure organizational commitment. Organizational commitment consists of 14 questions with 4 questions on affective commitment, 5 questions on continuance commitment, and 5 questions on normative commitment. In the reliability coefficient of the organizational commitment measurement tool, an organizational commitment was .87, an affective commitment was .77, a continuance commitment was .60, and a normative commitment was .77.

\subsection{Research procedure}

A preliminary survey was conducted against 15 school nutritionists from 1 April to 3 April 2019 to modify and supplement inappropriate questions. This survey was then distributed from 15 April to 30 April 2019 via email and institution visits. Questionnaires were collected from 108 school nutritionists for final analysis.

\subsection{Data analysis}

From the collected data, frequency, and percentiles were first calculated to examine the demographic characteristics of research subjects, and the reliability of the research tools was calculated using Cronbach's acoefficient. Correlation analysis was performed to investigate the correlation between variables, and the effect of each variable was examined through multiple regression analysis.

\subsection{Research results}

This study was conducted to investigate the relationship between self-efficacy and egoresilience perceived by nutritionists and organizational commitment, and the research results for each research question are as follows. 


\subsubsection{Correlation between the self-efficacy, ego-resilience, and organizational commitment of school nutritionists}

The results of analyzing the relationship between school nutritionists' self-efficacy, egoresilience, and organizational commitment are shown in [Table 1].

Table 1. Correlation between self-efficacy, ego-resilience, and organizational commitment

\begin{tabular}{|c|c|c|c|}
\hline Variable & Self-efficacy & Ego-resilience & Organizational commitment \\
\hline Self-efficacy & 1 & & \\
\hline Ego-resilience & $.23^{* *}$ & 1 & 1 \\
\hline Organizational commitment & $.60^{* *}$ & $.45^{* *}$ & 3.51 \\
\hline $\mathrm{M}$ & 3.38 & 3.71 & .58 \\
\hline SD & .76 & .54 & \\
\hline$* * p<.01$
\end{tabular}

The correlation coefficient between self-efficacy and organizational commitment was $\mathrm{r}=.60(\mathrm{p}<.01)$, and the correlation coefficient between ego-resilience and organizational commitment was $\mathrm{r}=.45(\mathrm{p}<.01)$. The correlation coefficient between self-efficacy and egoresilience was $\mathrm{r}=.23(\mathrm{p}<.01)$. Significant positive correlations were found between all variables.

\subsubsection{Effect of school nutritionists' self-efficacy and ego-resilience on organizational commitment}

Stepwise regression analysis was performed to examine the effect of school nutritionists' self-efficacy and ego-resilience on organizational commitment. Before conducting the regression analysis, the Durbin-Watson value, confirming the independence of residual, was 1.84 , which is close to 2 . The result of the VIF value, confirming multi-collinearity, was $1.00 \sim 1.09$, which is less than 10. The results of the stepwise regression analysis are shown in [Table 2].

Table 2 Relative effects between self-efficacy, ego-resilience, and organizational commitment

\begin{tabular}{|c|c|c|c|c|c|c|c|}
\hline Model & $\begin{array}{l}\text { Independent } \\
\text { variable }\end{array}$ & B & $\beta$ & $\mathrm{t}$ & $\mathrm{R} 2$ & R2 change & $\mathrm{F}$ \\
\hline \multirow{2}{*}{1} & (Constant) & 1.95 & & 13.29 & & & \multirow{2}{*}{$119.08^{* * * *}$} \\
\hline & Self-efficacy & .46 & .60 & $10.91^{* * *}$ & .36 & .36 & \\
\hline \multirow{3}{*}{2} & (Constant) & .82 & & 3.66 & & & \multirow{3}{*}{$90.94^{* * *}$} \\
\hline & Self-efficacy & .40 & .52 & $10.09^{* * *}$ & \multirow{2}{*}{.46} & \multirow{2}{*}{.10} & \\
\hline & Ego-resilience & .36 & .33 & $6.38^{* * * *}$ & & & \\
\hline
\end{tabular}

As shown in the results in [Table 2], in model 1 , the effect of self-efficacy $(\beta=.60)$ on organizational commitment was $36 \%$, and when comparing the self-efficacy $(\beta=.52)$ and egoresilience $(\beta=.33)$ with the standardized coefficient, self-efficacy had the largest effect. This additional model 2 showed an explanatory power of $46 \%$, which was an increase of $10 \%$. 


\section{Conclusion}

The purpose of this study was to verify the relationship between school nutritionists' selfefficacy, ego-resilience, and organizational commitment, and to analyze the effect of selfefficacy and ego-resilience on organizational commitment. A discussion of the result of this study focused on the research questions, is as follows.

First, upon analyzing the correlation between school nutritionists' self-efficacy and organizational commitment, there was a significant positive correlation between self-efficacy and organizational commitment. [10] analyzed the relationship between teachers' selfefficacy and empowerment to organizational commitment, where self-efficacy was found to have a significant positive relationship with organizational commitment. Such results demonstrate that, unlike other educational institutions or organizations, organizations with only a small number of members are characterized by intimate bonds between the members, and the role of self-efficacy is very important in leading the organization as it exerts its strengths through values and educational philosophies [21]. In addition, school nutritionists' ego-resilience and organizational commitment were found to have a significant positive correlation, and this implies that the higher the ego-resilience, the higher the perception of organizational commitment. These results are consistent with the study of [22], which analyzed the effect of teachers' ego-resilience and organizational commitment on happiness. Teachers' ego-resilience allows them to assume positive attitudes that are appropriate for different stress factors in their working environment, cope well with impulsive or chronic stress situations, and recover quickly from trauma [23][24]. This then enables them to overcome problems related to their organization as ego-resilience directly affects their desire for positive resolution [17][25].

Second, upon investigating the effect of school nutritionists' self-efficacy and egoresilience on their organizational commitment, self-efficacy was found to have a direct effect on organizational commitment with an explanatory power of $36 \%$. Nutritionists with high self-efficacy recognize the value of their members and inspire creativity to induct voluntary participation and devotion of the members, and increase organizational effectiveness by improving the sense of calling and responsibility [26][27]. These results will increase the nutritionists' emotional attachment to the organization, increase their sense of calling, as well as improving the level of organizational commitment. Organizational commitment was improved even further with the addition of ego-resilience in the inner and psychological aspects of the nutritionist, along with their self-efficacy. These results support the existing studies [13][25] on ego-resilience and organizational commitment. Existing studies argue that teachers with high ego-resilience have a tendency to feel more confident and competent in various problem situations or complex interpersonal relationships [28], which leads to excellent problem-solving and communication skills below and directly affects the level of adaptability and commitment below. Such studies provide a solid basis that explains the results of this study where ego-resilience and organizational commitment have significant effects. Given that nutritionists with high ego-resilience can adapt to and control stressful situations they face, it can be expected that nutritionists with high ego-resilience will have a greater level of coping to understand the stress as a challenge, rather than perceiving it negatively and control it positively, even if they encounter difficulties due to simultaneous demands, which is a characteristic common for their job.

Such results examine the effect of school nutritionists' self-efficacy and ego-resilience on organizational commitment, and the organizational commitment of the nutritionist is considered to be an important factor in exhibiting expertise to support nutritional education 
and healthy school life for students. This study is significant in that it identifies relationships between various variables to increase organizational commitment, and provides implications of social and political directions to develop plans to support this.

Based on the results of this study, the following limitations can be suggested.

First, research subjects in this study were limited to $\mathrm{K}$ and $\mathrm{U}$ cities, so there is a limit to generalizing its results to cover nutritionists across the country, and subsequent studies will be required for a more in-depth investigation into the organizational commitment of nutritionists across Korea.

Third, subsequent studies are necessary to analyze organizational commitment, considering the organizational environment, the characteristics and factors of the members, and the distinctiveness of schools in special situations; and to investigate the effects and relationship of other variables to support positive organizational commitment.

\section{References}

[1] Se-Woen Kim, "The effect of nutrition education on the dietary intake behavior and nutrition knowledge of adolescents," M.S. thesis, Hanyang University, (2016).

[2] Weaver. C. M., "The period of dramatic bone growth," Endocrine, vol.17, pp. 43-48, (2002)

[3] Pok-Ja Ku and Kyoung-Ae Lee, "A survey on dietary habit and nutritional knowledge for elementary school children's nutritional education,” Journal of the Korean Society of Dietary Culture, vol.15, no.3, pp.201-213, (2000)

[4] Seung Kwon Ki, "The management of the role conflicts in the member of schools," M.S. thesis, Kumoh National Institute of Technology, (2009)

[5] Mee Kim, Nam Hee Hong, "The effects of role conflict and self-efficacy on job satisfaction for hospital coordinators," Korean Journal of Hospital Management, vol.14, no.3, pp.104-131, (2009)

[6] Ha Yeong Min, "The Relationship between the Surface/Deep Acting in Emotion Labor and the Collectivism on the Organizational Commitment of Kindergarten and Childcare Teachers," The Korean Journal of Child Education, vol.31, no.5, pp.17-30, (2010)

[7] Dong-Hyun Park., "A study on the effects of daycare teacher's self-leadership on job satisfaction and organizational commitment," M.S. thesis, Chong-Shin University, (2013)

[8] Sheldon M. E., "Investments and involvements as mechanisms producing commitment to the organization," Administrative Science Quarterly, vol.16, no.2, pp.143-150

[9] Kwang-Hyuk Ko., “A study on the relationship among principal's servant leadership, teacher's efficacy, school organizational commitment, and school organizational effectiveness," M.S. thesis, Inha University, (2011)

[10] [Hyun Go., "The influence of director`s servant leadership on relation to empowerment and organizational commitment of childcare teachers," Journal of Early Childhood Education, vol.35, no.1, pp.5-28, (2015)

[11] Young Eun Kim., "The influence of child care teachers' emotional intelligence upon organizational commitment -focused on level 2 promotion subjects," Journal of Early Childhood Education \& Educare Welfare, vol.17, no.3, pp.56-74, (2013)

[12] Potter S. L., "Relationships between educator's organizational commitment, job, satisfaction, and administer's gender," Ph.D. dissertation, University of Liberty, VA, (2012)

[13] Seung-Hyun Oh and Yeon-Hee Kwon, “Structural correlations among director's servant leadership, teacher's ego-resilience and organizational commitment in the early childhood education center," Journal of Korean Child Care and Education, vol.13, no.1, pp.1-17, (2017)

[14] Hyeyoon Choi, "Servant leadership of directors and influence of collaborative organizational culture for the organizational commitment of teachers in kindergarten," Journal of Future Early Childhood Education, vol.22, no.4, pp.301-322, (2015) 
[15] Eun-Hee Kim and Hwie-Seo Park, "The impacts of organizational job stress factors on organizational commitment - focusing on the effects of ego-resilience," The Korean Association for Governance, vol.17, no.1, pp.1-28, (2010)

[16] Tai Hyong Moon, "Relations between early childhood educator's self-resilience, social intelligence, and organizational commitment," The Korean Journal of Child Education, vol.22, no.1, pp.5-19, (2013)

[17] Jeong-Mi Lee, Kyu Soo Kim. A Structural Model Analysis of Variables Affecting Organizational Commitment of Childcare Teachers. Korean Open Association for Early Childhood Education, vol.21, no.6, pp.83-109, (2016)

[18] Nam-Jin Kim, "A study on teacher-efficacy of elementary school special class teachers based on the background," The Journal of Special Children Education, vol.8, no.1, pp.59-76, (2006)

[19] Hyun-A Jung, "The effects of childcare teachers' emotional labor and ego-resilience on burnout," M.S. thesis, Kookmin University, (2014)

[20] Soo-Jung Go, "The determinants of job satisfaction and organizational commitment for the social welfare professionals," M.S. thesis, Yeungnam University, (2005)

[21] Yong Hak Kim, "Servant leadership and organizational commitment: the roles of empowerment and trust in leader," M.S. thesis, Yeungnam University, (2010)

[22] Mi Jin Kim, Byung Man Kim, "The mediating effect of organizational commitment on the relationship between ego resilience and happiness in early childhood teachers," The Korean Society for the Study of Child Education, vol.23, no.4, pp.143-162, (2014)

[23] In-Suk Kim, "Effect that leader-member exchange, ego resilience, organizational culture according to teachers' followership types have on job satisfaction," M.S. thesis, Daegu University, (2009)

[24] Masten A. S., Hubbard J., Gest S., Tellgen A., Garmwzy N., and Ramirez M., "Competence in the context of adversity: Pathways to resilience and maladaptation from childhood to late adolescence," Development and Psychopathology, vol.11, pp.143-169, (1990)

[25] Kang Seung Ji and Shon Yoo Jin, “A study on the relationship between teacher's commitment, organizational cultures, organizational commitment, and ego-resilience,” Journal of Early Childhood Education, vol.37, no.5, pp.317-337, (2017)

[26] Hardin F, "Impacting Texas public schools through a student servant-leader model: A case study," M.S. thesis, Texas Tech University, (2003)

[27] Whestone J. T., "Personalism and moral leadership: the servant leader with a transforming vision," Business Ethics: A European Review, vol.11, no.4, pp.385-392, (2002)

[28] Kirkman B. and Rosen B., "Beyond self-management: Antecedents and consequences of team empowerment," Academy of Management Journal, vol.42, no.1, pp. 58-74 
This page is empty by intention. 